\title{
Evaluation of a Histoplasma antigen Lateral Flow Assay for the rapid diagnosis of progressive disseminated histoplasmosis in AJDS patients
}

\author{
Diego H. Caceres ${ }^{1,2}$, Melissa C. Minderman³, Logan Cheney³, Beatriz L. Gomez ${ }^{4}$, L. Joseph Wheat ${ }^{3}$, Tom Chiller ${ }^{1}$, Mark Lindsley \\ 1. Centers for Disease Control and Prevention (CDC), Atlanta, GA, USA. 2. Oak Ridge Institute for Science and Education (ORISE), Oak Ridge, Tennessee, USA. \\ 3. MiraVista Diagnostics, Indianapolis, IN, USA. 4. Universidad del Rosario, Bogota, Colombia.
}

\section{INTRODUCTION}

Histoplasmosis is an important cause of mortality in persons living with HIV and AIDS (PLWHA), especially in countries where patients have limited access to antiretroviral therapies and diagnostic testing. In PLWHA, the progressive disseminated form of histoplasmosis (PDH) can be fatal without treatment. Early diagnosis is critical for providing proper treatment, however, current diagnosis by culture can take weeks and serology may be falsely negative early in infection or as a result of immunosuppression in these patients. Detection of Histoplasma antigen in patient specimens improves sensitivity and timeliness of diagnosis, but the current method by enzyme immunoassay must be performed by highly trained personnel in specialty laboratories. Recently, the development of the lateral flow technology has provided a method that is simple to use and can be performed in a setting closer to the patient. In this study, a lateral flow assay (LFA) was evaluated for the detection of Histoplasma antigen in sera.

\section{METHODS}

Using a MiraVista Diagnostics LFA for detection of Histoplasma antigen, we evaluated 47 serum samples: 19 from patients with AIDS and culture-proven PDH and 28 from patients with other fungal and bacterial infections, as well as healthy people (Figure 1). LFA devices were read by human eye and automated reader.

Study subjects and urine samples analyzed

Colombia serum samples $(n=43)$

Histoplasmosis $(n=19)$

Mycobacterium disease $(n=10)$

Cryptococcosis $(n=5)$

Pneumocystis pneumonia $(\mathrm{n}=1)$

Paracoccidioidomycosis $(n=2)$

Aspergillosis $(n=1)$

Candidiasis $(n=1)$

Salmonella disease $(n=2)$

Toxoplasmosis $(n=2)$

Addition baseline serum controls ( $n=4)$ Healthy individuals resident in endemic area

Baseline serum tested ( $n=47$ )

19 histoplasmosis cases

28 non histoplasmosis cases

\section{RESULTS}

When the LFA test was read by human eye, sensitivity was $95 \%$ and specificity was $82 \%$. Using an automated reader, sensitivity was $89 \%$ and specificity was $89 \%$ (Table 1 ). The Kappa index compared human eye and automated reader was 0.87 (Table 2). Crossreactions were observed principally in samples from patients with proven diagnosis of paracoccidioidomycosis.

\begin{tabular}{|c|c|c|c|c|c|c|}
\hline \multicolumn{7}{|c|}{ Table 1} \\
\hline & \multicolumn{3}{|c|}{ Human eye } & \multicolumn{3}{|c|}{ Auto-reader } \\
\hline & \multicolumn{3}{|c|}{ Culture } & & \multicolumn{2}{|c|}{ Culture } \\
\hline & & + & - & & + & - \\
\hline \multirow{2}{*}{ Mvista LFA } & + & 18 & 5 & + & 17 & 3 \\
\hline & - & 1 & 23 & - & 2 & 25 \\
\hline
\end{tabular}

Table 1: MiraVista Diagnostics Histoplasma Ag LFA analytical performance by human eye and automated reader.

Table 2: MiraVista Diagnostics Histoplasma Ag LFA concordance analysis between human eye and automated reader.

\begin{tabular}{c|c|c|c|}
\hline \multicolumn{3}{c}{ Table 2 } \\
\hline \multirow{1}{*}{ Visual } & \multicolumn{3}{c}{ Automated reader } \\
\cline { 2 - 4 } & & + & - \\
\cline { 2 - 4 } & + & 20 & 3 \\
\cline { 2 - 4 } Kappa & - & 0 & 24 \\
\hline \multirow{3}{*}{} & \multicolumn{3}{|c|}{$\mathbf{0 . 8 7}(\mathbf{0 . 7 4 - 1 . 0 0 )}$} \\
\hline
\end{tabular}

\section{CONCLUSIONS}

The MiraVista Diagnostics LFA had high analytical performance and good agreement between human eye and automated reader. This LFA allows Histoplasma antigen testing with minimal laboratory equipment and infrastructure requirements. Based on these results, this new method is a viable option for rapid diagnosis of PDH. LFA provides highly sensitive results in $<1$ hour, being faster and more sensitive for PDH than other immunological assays, such as ELISA (three to five hours; $>90 \%$ sensitivity), immunodiffusion and complement fixation (two days; $\sim 65 \%$ sensitivity), and conventional microbiological tests, including histopathologic examination (one to two days; $~ 75 \%$ sensitivity) and culture (two to four weeks; $~ 80 \%$ sensitivity). Prompt diagnosis of PDH could impact public health by initiating early treatment thereby reducing mortality.

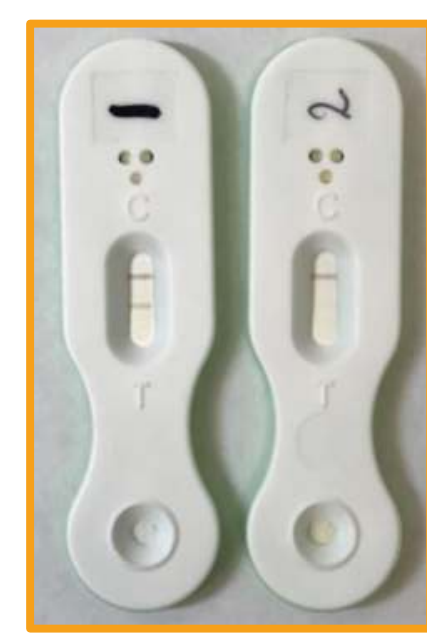

1. Positive LFA 2. Negative LFA

Contact: Diego H. Caceres, BSc-MSc. Mycotic Diseases Branch. Centers for Disease Control and Prevention (CDC)

xju7@cdc.gov 